\title{
Marco conceptual de los Estilos de Aprendizaje. El caso de los estudiantes en formación inicial de profesores de química Conceptual framework of learning styles. The case of students in initial training of chemistry teachers
}

\author{
Michal Elías Godoy ${ }^{1}$, Flavio Zamorano Pontiggia ${ }^{2}$, Javiera Pérez Cáceres ${ }^{3}$, Marijana Tomljenovic Niksic ${ }^{4}$ y Elisa \\ Zúñiga Garay ${ }^{5}$ \\ michal.elias@umce.cl1, vincenzo.zp.flavio@gmail.com², javieracaceres13@gmail.com³ ${ }^{3}$ marijana.tomljenovic@umce.cl ${ }^{4}$, \\ elisa.zuniga@umce.cl ${ }^{5}$
}

Departamento de Química de la Facultad de Ciencias Básicas

Universidad Metropolitana de Ciencias de la Educación

Santiago, Chile

\begin{abstract}
Resumen- El conocer cómo les gustaría aprender a los o las estudiantes permitiría utilizar medios educativos motivantes y contribuir al desarrollo de competencias comprometidas en su Perfil de Egreso. Este trabajo tiene como objetivo el determinar los Estilos de Aprendizaje (EA) que declaran los y las estudiantes en formación de Profesor de Química. Se elaboró un marco conceptual acerca de los EA, a través de una revisión bibliográfica regulada por los criterios de CorrelaciónContemporaneidad-Sustancialidad. Se presenta un marco conceptual con una evaluación histórica y dialógica, a partir de los grandes aportes en esta temática (Aristóteles, Vygotsky, Piaget, Kolb, Fleming y Gardner). La determinación de los EA se realizó desde un paradigma mixto, con un diseño sistemático en torno a una metodología cuasiexperimental, con dos grupos naturalmente constituidos de estudiantes (cohortes 2020 y 2021), aplicando el Cuestionario Honey-Alonso de los Estilos de Aprendizaje (CHAEA). Las respuestas del cuestionario evidenciaron una alta consistencia interna (coeficiente $\alpha$ de Cronbach entre 0,8 y 0,9$)$ y una varianza media entre 0,7 y 1,1 . Se observó un perfil mayoritariamente Asimilador, luego Divergente y Acomodador, además de evidenciar entre un $20 \%$ y $10 \%$ de preferencias con perfiles mezclados, en todos los casos no se observaron diferencias de género.
\end{abstract}

\section{Palabras clave: estilos de aprendizaje, estudiantes en formación inicial, pedagogía en química}

Abstract- Knowing student's preference in how to learn would allow
them to use motivating educational means and contribute to
development of competencies committed in their Graduate Profile.
The objective of this work is to determine the Learning Styles (LS) that
students in Chemistry Teacher training declare. A conceptual
framework to AE was developed through a bibliographic review
following by the criteria of Correlation, Contemporaneity
Substantiality. A conceptual framework is presented with a historical
and dialogical evaluation of great contributions (Aristotle, Vygotsky,
Piaget, Kolb, Fleming, and Gardner). The determination of LS was
carried out from a mixed paradigm, with a systematic design around a
quasi-experimental methodology, with a naturally constituted groups
of students (cohorts 2020 y 2021), applying the Honey-Alonso
Learning Styles Questionnaire. The responses to the questionnaire
showed high internal consistency (Cronbach's $\alpha$ coefficient between
0.8 and 0.9) and a mean variance between 0.7 and 0.1 . A mostly
Assimilatory profile is exhibited, then Divergent and Accommodator
type, in addition to showing between $20 \%$ and $10 \%$ of preferences with
mixed profiles, in all cases they were not observed gender differences.
Keywords: learning styles, initial training students, chemistry pedagogy

\section{INTRODUCCIÓN}

La Pandemia del COVID 19 nos ha dejado más de 4,4 millones de muertos y 215 millones de casos confirmados en 200 países (BBC, 2021), aumentando las desigualdades sociales y educativas (Quiroz, 2020). Los cierres de establecimientos educativos para evitar fuentes de contagio han hecho que personas de todos los grupos etarios hayan visto afectados sus procesos de enseñanza aprendizaje, lo anterior llevó a adoptar clases on line o vía plataformas virtuales (Varguillas y Bravo, 2020), dentro de lo posible.

Los estudiantes que actualmente están en formación tienen un marcado uso de las tecnologías y manejo de medios digitales para aprender o para comunicarse (Elías, Tomljenovic y Zúñiga, 2021). Sus formas de comunicación y el uso de medios digitales hacen necesario considerar desde las didácticas sus preferencias en los procesos de enseñanza aprendizaje y más aún en el aprendizaje en ciencias naturales y en contexto de Pandemia.

Debido a la propia individualidad humana, nadie puede asegurar que aprende de manera idéntica; sin embargo, existen semejanzas en cómo se aprende y se entiende el mundo. De esta forma, los EA se refieren a cómo los y las estudiantes aprenden o les gustaría aprender.

Si hablamos de los EA, los Millennials (1990-1999) son pragmáticos y realizan múltiples tareas con una corta capacidad de atención, los procesos de enseñanza aprendizaje deberían responder en este caso a presentar la información en tiempos cortos, de manera de obtener su atención, considerando que tienden a distraerse fácilmente. A su vez los Post-Millennials (2000-2009) prefieren alternativas educativas donde el paso a paso como por ejemplo a través de tutoriales de YouTube es común en sus procesos de enseñanza aprendizaje y en el ámbito profesional, buscan lugares de interés donde existan oportunidades de capacitación y de desarrollo, en cuanto a la forma en que prefieren aprender, destacan la observación y el hacer por sobre la lectura y la observación (Elías et al., 2021). 
Ambas generaciones muestran preferencias generacionales de cómo aprender, y a eso hay que sumar que como individuos también presentan diferencias.

El CHAEA permite discernir entre cuatro estilos de aprendizaje: experiencia concreta o aprendizaje pragmático, conceptualización abstracta o teórica, experimentación activa o aprendizaje a través de la acción y observación reflexiva o enfoque múltiple previo a la toma de decisiones. A su vez, estas cuatro formas se combinan para dar cuatro tipos de estudiantes: Convergentes que enfatizan el método deductivo, Divergentes que usan enfoques desde múltiples perspectivas antes de actuar, Asimiladores que prefieren el abordaje inductivo y Acomodadores que optan por experimentar haciendo (Kolb y Kolb, 2017; Borracci y Arribalzaga, 2015). El conocimiento desarrollado en este trabajo podría dar a los y las profesores/as herramientas que les permitan adecuarse a sus estudiantes $\mathrm{y}$, a estos últimos, el aprender de manera más consciente con el fin de ser un individuo más responsable que se apropie de su proceso educativo.

\section{CONTEXTO}

En los tiempos de pandemia, el estrés y la modalidad on line, demanda conocer las preferencias en los EA que las y los estudiantes declaran como un medio orientador en el diseño metodológico de contenidos, además de ser un instrumento facilitador en sus propios procesos de aprendizaje.

Para este estudio se planteó el objetivo de levantar un marco conceptual en torno a los EA, a través de una revisión bibliográfica, y caracterizar mediante el CHAEA los estilos de aprendizaje que declaran los y las estudiantes de primer semestre de las cohortes 2020 y 2021 de Pedagogía en Química de una universidad estatal chilena.

\section{DESCRIPCIÓN}

El levantamiento del marco conceptual se llevó a cabo a través de una revisión bibliográfica, con el propósito de establecer relaciones, para la construcción del concepto de "Estilos de Aprendizaje". Se realizó una pre-búsqueda, utilizando los motores de búsqueda y bases de datos: Google, Google Académico, Dialnet, Elsevier y SciELO, representando en nubes de palabras las palabras claves utilizadas, se utilizó la plataforma gratuita Infogram, donde el tamaño de la palabra indica la frecuencia de la presencia en los resúmenes de los artículos. Como criterio de exclusión se consideró la repetición de artículos entre los motores de búsqueda utilizados. Las búsquedas se realizaron tanto en español como en inglés.

Para la construcción del marco conceptual se utilizaron los siguientes criterios jerarquizados: la Correlación, la que permite establecer el vínculo existente entre la temática a conceptualizar (Estilos de Aprendizaje) y los posibles aportes que permitan la construcción del concepto en sí (teniendo como parámetro la semántica del concepto); la Contemporaneidad, que delimita y prioriza los artículos recientes (publicaciones en los últimos 5 años), pero manteniendo artículos fundantes o esenciales de las ideas, ya que independiente del tiempo transcurrido siguen siendo fruto de nuevas reflexiones y profundizaciones; y la Sustancialidad, que determina lo que sería un aporte a la conceptualización. Finalmente, las palabras claves utilizadas fueron: estilos de aprendizaje, aprendizaje, inteligencia, inteligencias múltiples, memoria y aprendizaje, procesamiento de información, aprendizaje autodirigido, VARK y educación.

Para la caracterización de las preferencias en los EA se utilizó un diseño metodológico cuasi-experimental, sin grupo control y un grupo naturalmente constituido de estudiantes de primer semestre de Pedagogía en Química de una universidad estatal chilena, a los cuales se les aplicó el CHAEA en la forma de Formularios de Google, como instrumento de recolección de información. El cuestionario se aplicó durante el primer semestre del año $2020(n=25)$ y $2021(n=10)$, en el marco de una asignatura regular.

La investigación se llevó a cabo bajo un paradigma constructivista sociocultural (Córdoba, 2020) - positivista, de metodología cuantitativa. La primera sección se refirió a caracterizar a los o las estudiantes, previa aceptación de un Consentimiento Informado, donde se registraron datos personales de los participantes (su nickname o alias, su género y su edad). Finalmente, se presentaron las instrucciones para responder el cuestionario, el que constaba de 80 afirmaciones estructuradas y una escala de Likert de 4 puntos, siendo 1 "muy en desacuerdo" y 4 "muy de acuerdo". La confiabilidad se determinó a través del Coeficiente $\alpha$ de Cronbach y los estadísticos descriptivos de la muestra por el software IBM SPSS Statistics 25

Para el análisis de los resultados del CHAEA se elaboró una matriz con los cuatro cuadrantes de los EA, considerando 4 zonas en la forma de círculos concéntricos, siendo la zona interna la que comprende a quienes no presentan una preferencia definida con respecto a los EA y la zona externa a quienes la preferencia se encuentra más definida.

\section{Resultados}

La revisión bibliográfica utilizó las palabras claves seleccionadas conforme a la semántica o contexto de los EA que, fueran englobando otros conceptos; por ejemplo, Inteligencias Múltiples considera a Inteligencia Emocional. Además, se consideró el que permitan conducir hacia enfoques o perspectivas psicológicas/educativas o evolutivas/anatómicas de los EA; por ejemplo, se observa que las palabras claves presentes en las nubes de palabras (Figura 1), como Tecnología o Ética, resultan elementos que no ofrecen un mayor aporte para el Marco Conceptual, pues solo se remiten al medio educativo o criterios sociales.

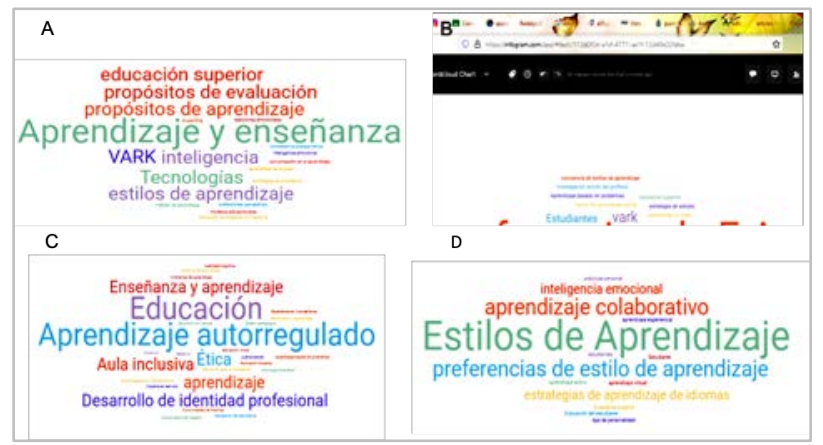

Figura 1. Nubes de palabras claves presentadas en diferentes artículos recuperados desde los motores de búsqueda: A) SciELO, B) Google Académico, C) Elsevier y D) Dialnet. 


\section{A. Marco Conceptual en torno a los Estilos de Aprendizaje}

El aprendizaje está fuertemente ligado al concepto de inteligencia. Kosherbayeva, Abdreimova, Kosherba, y Anuarbek, (2013) la describen como "la capacidad del individuo para empatizar, para contribuir, a la capacidad de la actividad dialógica activa en el nivel de asociación y cooperación" (p.886). Por su parte, Goldstein, Princiotta y Naglieri (2015) plantean a la inteligencia como un rasgo evolutivo más allá de la capacidad racional, ya que la inteligencia permite la resolución de problemas, incluyendo la adaptación intencionada $\mathrm{y}$, en el ser humano, el razonamiento (Ardila, 2011).

De Azcárate (1873) rememora una de las primeras definiciones de inteligencia a partir de lo mencionado por Aristóteles, donde hace referencia a lo que no puede ser, atribuye la inteligencia a algo valorativo, crítico y que nos permite discernir; sin embargo, esta mirada "humaniza" o da una mirada antropocéntrica a la inteligencia. Gardner (2016) amplía el entendimiento de las manifestaciones de la inteligencia y cómo esta puede también estar presente de variadas formas en humanos, reconociendo que también está presente, en mayor o menor grado, en otros tipos de animales, alejándonos de las ideas antropocentristas iniciales.

La inteligencia desde la perspectiva evolutiva (Goldstein, Princiotta y Naglieri, 2015) considera el desarrollo de órganos específicos que permiten adquirir información del exterior a un organismo y estar "preparados" para el ambiente. Así, por ejemplo, Lamb (2011) expone que el desarrollo de un órgano que permita distinguir entre el día y la noche, permitiría a diferentes especies poder estar preparadas para esconderse o salir a buscar comida; sin embargo, la capacidad de distinguir objetos, o de desarrollar percepciones de profundidad, como operan actualmente los ojos humanos, darían pie al desarrollo de nuevas actividades, pues la información adquirida también sería mucho mayor que al inicio, mejorando cómo se procesa la información (Baker et al., 2014).

El cerebro procesa la información transmitida por los sentidos (van Kesteren y Meeter, 2020), desde una mirada más holística, sería la mente y su propia singularidad la capaz de dar forma a esta información, mediante una serie de herramientas, como la memoria y el aprendizaje. Squire, Genzel, Wixted y Morris (2015) consideran a la memoria como la capacidad de retener la información adquirida mediante los diferentes sentidos, almacenándola en diferentes zonas del cerebro, donde solo los aprendizajes se expresarían (Kazdin, 2000).

El aprendizaje se relaciona con la memoria, como parte de las herramientas presentes en los procesos de enseñanza aprendizaje, con repercusiones positivas o negativas, como el estrés y la presión ejercida en un ambiente social (Vogel y Schwabe, 2016). También la intencionalidad se relaciona con el aprendizaje, a diferencia de la memoria la que no requiere de una intencionalidad (Moreno, Romero, Salazar y Ortega, 2016). Para que ocurra un aprendizaje debe existir motivación o intención (Al-Hoorie y Dornyei, 2019); por ejemplo, para adquirir una habilidad o un saber inmediato, ese requiere la memoria y en particular, la memoria implícita (Moreno et al., 2016), mientras que el conocimiento empírico modifica el comportamiento del individuo y/o el desarrollo de este saber prolongado en el tiempo, haciendo referencia al proceso de aprender como la transformación de una conducta de manera continua en el tiempo, fruto de la propia experiencia (Aguilar, Espinoza, Oruro y Carrión, 2010).
Por lo tanto, el pasar de un acto inconsciente como memorizar, a uno más voluntario como el aprender, hace de los seres humanos organismos cognitivamente más desarrollados y con capacidad de razonar, adaptarse al ambiente y solucionar problemas, cualidades que les permiten a las especies sobrevivir a los cambios de la naturaleza (Ardila, 2011).

En términos fenomenológicos, el aprender aparenta ser más "real", o al menos ser una experiencia más consciente, que la propia inteligencia (Jensen, 1989), dado que la naturaleza del aprendizaje, es un proceso que se presenta de manera más empírica, medible y consciente (Harris y Pressley, 1991), relevando el estudiar cómo una forma de aprender. Dado que la memoria y la inteligencia son productos del aprendizaje, este último sería una especie de sello personal de cómo se construye el "propio mundo del conocimiento", desde la propia "individualidad" traduciéndose en Estilos de Aprendizaje.

\section{B. Estilos de Aprendizaje}

Desde la investigación en psicología, las diferencias individuales de cada persona se han definido como "estilos": estilos cognitivos, estilos de aprendizaje, estilos de resolución de problemas, estilos de pensamiento, estilos de toma de decisiones, entre otros (Woolfolk, 2014). La palabra estilo proviene del latín stylu que significa carácter, peculiaridad, modo, manera o forma de hacer las cosas y se utiliza en la vida cotidiana para aludir a alguna cualidad distintiva y propia de una persona o grupo de personas en diferentes esferas de la actividad humana (Vox, 1991, citado en Aguilera y Ortiz, 2009).

Existen distintos modelos de EA que han sido aceptados por la comunidad especializada; como por ejemplo, el sistema de representación del modelo PNL (Programación Neurolingüística), el modo de procesar la información, la categoría bipolar, las preferencias de pensamiento, desarrollo de las capacidades, el modelo de las inteligencias múltiples de Gardner, entre otros (Aragón y Jiménez, 2009; Romero, Romero y Barboza, 2021

Una definición de los EA se le atribuye a Aristóteles, en el año 334 a.C., donde se señala que "cada niño posee habilidades y talentos específicos" (Reiff, 1992, p.7), por lo que cada persona que está comenzando a aprender algo nuevo debe considerar que es único en su propia esencia y por lo tanto, posee sus propias fortalezas para el aprendizaje. Esta definición estructuró durante siglos el diseño de teorías con respecto al aprendizaje y a los propios EA.

En el siglo XX, el psicólogo ruso Lev Vygotsky consideró que el "ambiente social" jugaba un rol de suma relevancia en los procesos de aprendizaje por lo que, para lograr significar los elementos a aprender, es necesario que el espacio social donde se desenvuelve la persona sea apropiado para el desarrollo de las habilidades del o la estudiante (Vygotsky, 1979). Por otra parte, el epistemólogo y biólogo Jean Piaget, estableció el foco en el propio desarrollo del individuo, posicionando así un desarrollo cognitivo que era transversal a las culturas, y en consecuencia, el ambiente social quedó relegado a aspectos secundarios, donde el o la protagonista del aprendizaje fue el desarrollo del propio individuo, a través de la influencia de sus propias experiencias (Huitt y Hummel, 2003). La contemporaneidad de Vygotsky y Piaget, explica la similitud de sus perspectivas y miradas (Vielma y Salas., 2000; Raynaudo y Peralta, 2017; Barba, Cuenca y Rosa, 2007). 
Posteriormente, el psicólogo Howard Gardner contempló diferentes formas y estilos para poder desarrollar los saberes y conocimientos, desarrollando así la Teoría de las Inteligencias Múltiples (Gardner, 2016). Luego, Neil Fleming diseñó un cuestionario específico para los perfiles de los EA, llamado cuestionario VARK, por su sigla que se refiere a la traducción del inglés de Visual, Aural/Auditory, Read/Write y Kinesthetic, es decir, lo visual, acústico, lecto-escritura y kinestésico; sin embargo, el mismo autor cuestiona si son "estilos" de aprendizaje y no "preferencias" de aprendizaje. Según Fleming (2012), los resultados del cuestionario VARK hacen referencia a un concepto diferente de los estilos de aprendizaje, puesto que los EA dan cuenta de un amplio abanico de conductas de aprendizaje, estableciendo en qué contextos les es mejor aprender a las personas, en qué momentos del día o la cantidad de personas participantes un proceso de aprendizaje. Por el contrario, el cuestionario se trataría "sobre las personas y su aprendizaje y se centra en las modalidades que podría preferir al aprender" (Fleming, 2012, p.1; Cabual, 2021), dejando el énfasis del cuestionario en el aprendizaje y no en la enseñanza.

Posteriormente, David Kolb establece que la experiencia remite a toda actividad que pueda permitir el aprender (Kolb y Kolb, 2013), transforma el foco del aprendizaje y se centra en lo que podría propiciar el aprendizaje en sí mismo, las actividades o comportamientos que permitirían el proceso de aprendizaje. En esta misma línea, Freedman y Stumof (1980) establecen que el aprendizaje depende de la experiencia vivida y tendría cuatro etapas: la experiencia concreta, la observación reflexiva, la conceptualización abstracta y la experimentación activa.

Aunque las etapas establecidas por Freedman y Stumof parecieran ser cruciales y todas necesarias durante el proceso de aprendizaje, en la práctica las personas poseen preferencias para la realización de sus actividades. Para el análisis de estas etapas, consideradas en el proceso de aprender, surge un diseño de lineamientos dicotómicos sobre la percepción (experiencias concretas-conceptualización abstracta) y el procesamiento de la información (experiencias activas-observación reflexiva), siendo el aprendizaje, el producto del cómo se perciben los fenómenos y cómo se procesan (Kolb, 2014).

Kolb propone un modelo de 4 cuadrantes, siendo el eje de las ordenadas los conceptos dicotómicos de la percepción y el eje de las abscisas los del procesamiento de la información (Rodríguez, 2017).

C. Determinación de los Estilos de Aprendizajes de los estudiantes de primer año de Pedagogía en Química

Las personas participantes de este estudio $(n=25$, cohorte 2020; $\mathrm{n}=10$, cohorte 2021) correspondieron al $92,59 \%-2020 \mathrm{y}$ al 100\%-2021 de los grupos naturalmente constituidos, con un rango etario entre 18 y 27 años (45,8\%-2020 y 40\%-2021 tenían 18 años), no observándose diferencias de género en la carrera de Pedagogía en Química, a diferencia de la brecha de género que se observa de manera transversal en otras carreras de las instituciones de Educación Superior, especialmente las relativas a las ciencia, tecnología y matemáticas (SIES, 2018).

Las respuestas del cuestionario aplicado establecieron una clara tendencia hacia un perfil asimilador (45,83\%-2020; 50\%$2021)$, seguido de un perfil divergente $(20,83 \%-2020 ; 20 \%$ 2021) y acomodador (12,5\%-2020; 20\%-2021), solo se observó un estudiante con un perfil convergente en la cohorte 2021. (Figura 2). Las respuestas muestran una alta consistencia interna ( $\alpha$ de Cronbach de 0,822-2020 y 0,959-2021) y una varianza $(\sigma)$ promedio de $0,72 \pm 0,262020$ y $1,11 \pm 0,50-2021$.

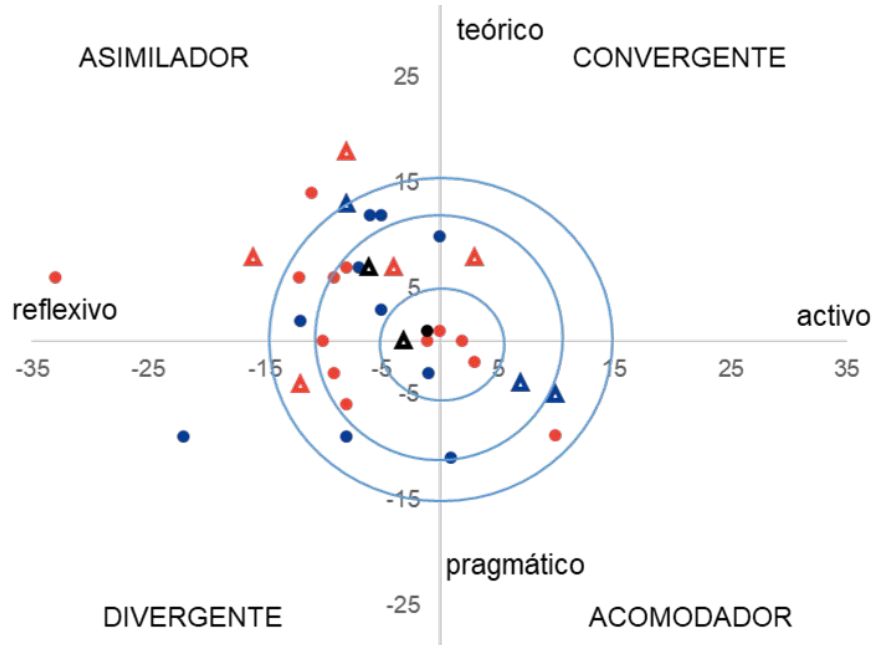

Figura 2. Matriz de los cuatro cuadrantes de los EA de Kolb, según CHAEA de los y las estudiantes de primer semestre de la carrera de Pedagogía en Química de las cohortes 2020 (círculos) y 2021 (triángulos), donde el color rojo identifica género femenino, el color azul género masculino y el color negro no identifica género.

En relación a las características de los y las estudiantes (palabras en minúscula en los ejes de la Figura 2), se observa una mayor densidad de los resultados en los cuadrantes que corresponden a sujetos más Reflexivos que Activos. Esta densidad de resultados, concentrados más en el eje de las ordenadas que el de las abscisas, nos indicaría también cuan definido es el EA, con respecto al par dicotómico de lo Pragmático-Teórico. Considerando la distribución a lo largo del eje de las ordenadas, se observó que los y las participantes manifiestan una tendencia marcada hacia lo Teórico, tendiendo a presentar preferencias Teórica-Reflexiva; sin embargo, la alta presencia de los resultados en el cuadrante Divergente, establece una segunda categoría, la cual apuntaría a una preferencia Pragmática-Reflexiva.

Se observó la presencia mayoritaria de un perfil/tipo de estudiante Asimilador, sin diferencia de género, de manera similar a lo observado en otras carreras del área científica. Borracci y Arribalzaga (2015), tras la aplicación del mismo cuestionario, observaron estos mismos perfiles en estudiantes con distintos niveles de progresión o situación académica, donde los o las estudiantes con preferencias TeóricasReflexivas habían finalizado su proceso académico y las y los estudiante con un perfil más neutral o indefinido habían desertado de la carrera. Por su parte, Gurpinar, Alimoglu, Mamakli y Aktekin (2010) sugieren métodos de enseñanza más tradicionales para estudiantes de medicina de primer año, ya que serían más efectivos en estudiantes con un perfil Asimilador, estas preferencias se asocian a la escolarización (educación formal $\mathrm{y}$ tradicional) antes del ingreso a la universidad.

Según Gutiérrez (2018), las estrategias de aprendizaje se deben orientar a cada par dicotómico expuesto por Kolb; por ejemplo, en sintonía con Gardner (1998) y con Gutiérrez (2018), para el perfil Asimilador predominante en los sujetos participantes de este estudio, se esperaría que enfrenten su aprendizaje desde un modelamiento teórico y utilicen medios 
educativos orientados a investigaciones, encausados a informes escritos, lectura de texto, organización de datos, así como la realización de discusiones y debates, especialmente en torno a temas de ciencia viva.

\section{CONCLUSIONES}

El marco conceptual en torno a los EA permitió reconocer conceptos y autores claves, así como sus aportes a esta temática. El CHAEA determinó que el perfil Asimilador es el preferente en los y las estudiantes de primer semestre de Pedagogía en Química, sin diferencia de género, para las cohortes 2020 y 2021. Los resultados obtenidos permiten dar precedentes de cómo los estudiantes quieren aprender, siendo de utilidad dentro de la propia planificación, tanto de los y las profesores/as como de los y las estudiantes, invitando a los primeros al diseño de los recursos o acciones educativas que permitan responder a las preferencias de sus estudiantes, y a estos últimos a ser más conscientes de sus propios procesos de enseñanza aprendizaje.

\section{AGRADECIMIENTOS}

Se agradece al Programa de Doctorado de la Universidad Metropolitana de Ciencias de la Educación que, mediante su programa de becas se hizo partícipe de este estudio.

\section{REFERENCIAS}

Aguilar, L., Espinoza, G., Oruro, E. y Carrión, D. (2010). Aprendizaje, Memoria y Neuroplasticidad. Temática Psicológica, 6(6), https://doi.org/10.33539/tematpsicol.2010.n6.856

Aguilera, E. y Ortíz, E. (2009). Las investigaciones sobre los estilos de aprendizaje y sus modelos explicativos. Revista Estilos de Aprendizaje, 4(4), 22-35.

Al-Hoorie, A. y Dornyei, Z. (2017). The Motivational Foundation of Learning Languages Other Than Global English: Theoretical Issues and Research Direction. Modern Language Journal, 101(3), 455-468. https://doi.org/10.1111/modl.12408

Aragón, M. y Jiménez, Y. (2009). Diagnóstico de los estilos de aprendizaje en los estudiantes: Estrategia docente para elevar la calidad educativa. CPU-e, Revista de Investigación Educativa, (9), 1-21. http://www.uv.mx/cpue/num8/opinion/aragon_estilos_ap rendizaje.html

Ardila, R. (2011). Inteligencia. ¿qué sabemos y qué nos falta por investigar? Revista de la Academia Colombiana de Ciencias Exactas, Físicas y Naturales, 35(134), 97-103. http://www.scielo.org.co/scielo.php?script=sci_arttext\& pid $=$ S0370-39082011000100009

Barba, M., Cuenca, M. y Rosa, A., (2007). Piaget y LS Vigotsky en el análisis de la relación entre educación y desarrollo. Revista Iberoamericana de Educación, 43(1), 1-12. https://doi.org/10.35362/rie4312341

Baker, C., Peterson, E., Pulos, S. y Kirkland, R., (2014). Eyes and IQ: A meta-analysis of the relationship between intelligence and "Reading the Mind in the Eyes". Intelligence, 44, 78-92. http://dx.doi.org/10.1016/j.intell.2014.03.001

BBC (30 de agosto 2021) The Visual and Data Journalism Team. Covid map: Coronavirus cases, deaths, vaccinations by country. BBC News. Recuperado de https:/www.bbc.com/news/world-51235105

Borracci, R. y Arribalzaga, E. (2015). Estilos de aprendizaje de Kolb en estudiantes de medicina. Medicina (Buenos Aires), 75(1), 73-80. http://www.medicinabuenosaires.com/revistas/vol7515/n2/73-80-Med75-1-6280-Borracci-A.pdf

Cabual, R.A. (2021) Learning Styles and Preferred Learning Modalities in the New Normal. Open Access Library Journal, 8, 1-14. doi: 10.4236/oalib.1107305.

Córdoba, M. (2020). El constructivismo sociocultural lingüístico como teoría pedagógica de soporte para los Estudios Generales. Nuevo Humanismo. 8(1), 96. https://doi.org/10.15359/rnh.8-1.4

De Azcárate, P. (15 de diciembre 2020). Aristóteles · Moral a Nicómaco. Proyecto Filosofía en español. Recuperado de http://www.filosofia.org/cla/ari/azc01166.htm

Elías, M., Zúñiga, E. y Tomljenovic, M. (2021).Desafíos del profesor de ciencias frente a estudiantes Millennials y Post-Millennials. Revista de Estudios y Experiencias en Educación. xx (x), x-x. (en prensa).

Fleming, N. (diciembre 2012). Facts, Fallacies and Myths: VARK and Learning Preferences. VARK-learn. Recuperado el 09 de enero 2021 de https://www.varklearn.com/wp-content/uploads/2014/08/Some-FactsAbout-VARK.pdf

Freedman, R. y Stumof, S. (1980). Learning Style Theory: Less than Meets the Eye. the Academy of Management Review, 5 https://doi.org/10.5465/amr.1980.4288873

(3), 445

Gardner, B. y Korth, S. (1998). A Framework for Learning to Work in Teams. Journal of Education for Business, 74(1), 31. https://doi.org/10.1080/08832329809601657

Gardner, H. (2016). IV. ¿QUÉ ES UNA INTELIGENCIA?, Estructuras de la mente: La teoría de las inteligencias múltiples. I. la idea de las inteligencias múltiples (60-62). Fondo de cultura económica. http://biblioteca.udgvirtual.udg.mx:8080/jspui/bitstream/ $123456789 / 593 / 1 /$ Estructura\%20de\%20la\%20mente. $\% 2$ 0teoria $\% 20 \mathrm{de} \% 201 \mathrm{las} \% 20$ Inteligencias\%20multiples.pdf

Goldstein, S., Princiotta, D., Naglieri, J. (2015). Part I: 1: The Evolution of Intelligence. Handbook of Intelligence, Springer, 3. https://doi.org/10.1007/978-1-4939-1562-0

Gurpinar, E., Alimoglu, M. K., Mamakli, S., y Aktekin, M. (2010). Can learning style predict student satisfaction with different instruction methods and academic achievement in medical education? Advances in Physiology Education, 34(4), 192-196. http://dx.doi.org/10.1152/advan.00075.2010

Gutiérrez, M. (2018). Estilos de aprendizaje, estrategias para enseñar. Su relación con el desarrollo emocional y “aprender a aprender". Tendencias Pedagógicas, 31(1), 83-96. http://dx.doi.org/10.15366/tp2018.31.004

Harris, K. y Pressley, M. (1991). The Nature of Cognitive Strategy Instruction: Interactive Strategy Construction.

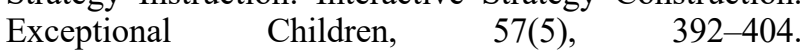
http://dx.doi.org/10.1177/001440299105700503 
Huitt, W. y Hummel, J. (19 de diciembre 2020). Piaget's theory of cognitive development. Educational Psychology Interactive. Recuperado de https://intranet.newriver.edu/images/stories/library/stenn ett_psychology_articles/Piagets\%20Theory\%20of\%20C ognitive\%20Development.pdf

Jensen, A. (1989). The relationship between learning and intelligence. Learning and Individual Differences, 1(1), 37-62. https://doi.org/10.1016/1041-6080(89)90009-5

Kazdin, A. (2000). Encyclopedia of Psychology. Oxford University

Press. http://www.indigenouspsych.org/Members/Churchill,\%2 0Scott/Churchill\%20\%20Encyclopedia\%20Articles\%20Phenom.pdf

Kolb, Alice Y. and Kolb, David A. (2017). Experiential Learning Theory as a Guide for Experiential Educators in Higher Education. Experiential Learning \& Teaching in Higher Education, 1(1), Article 7. Available at: https://nsuworks.nova.edu/elthe/vol1/iss1/7

Kolb, A. y Kolb, D. (2013). Experiential learning theory and individual learning styles, The Kolb Learning Style Inventory 4.0, A Comprehensive Guide to the Theory, Psychometrics, Research on Validity and Educational Applications (6-7). Experience Based Learning Systems. https://learningfromexperience.com/research-library/thekolb-learning-style-inventory-4-0/

Kolb, D., (2014). Experiential Learning: Experience as the Source of Learning and Development. Pearson Education. https://www.researchgate.net/publication/315793484_Ex periential_Learning_Experience_as_the_source_of_Lear ning_and_Development_Second_E_dition

Kosherbayeva, A., Abdreimova, K., Kosherba, G. y Anuarbek, A. (2013). Synthesis of Achievements of World Mankind İn Humanity Pedagogy. Procedia - Social and Behavioral Sciences, 89, 886-889. https://doi.org/10.1016/j.sbspro.2013.08.950

Lamb, T. (19 de diciembre 2020). La Evolución del Ojo. Investigación y Ciencia. Recuperado de https://www.investigacionyciencia.es/revistas/investigaci on-y-ciencia/la-fsica-de-la-inteligencia-533/la-evolucindel-ojo-9122

Moreno, J., Romero, M., Salazar, A. y Ortega, B. (2016). Estudio piloto. Memoria implícita, memoria explícita y deterioro cognitivo: evolución en el trastorno psicótico. Enfermería Global, $15 \quad$ (1), 135. http://scielo.isciii.es/scielo.php?script=sci_arttext\&pid=S 1695-61412016000100007

Quiroz, C. (2020). Pandemia Covid-19 e Inequidad Territorial: El Agravamiento de las Desigualdades Educativas en Chile. Revista Internacional de Educación para la Justicia Social,

$9(3 \mathrm{e})$. https://revistas.uam.es/riejs/article/view/12143

Raynaudo, G. y Peralta, O. (2017). Cambio conceptual: una mirada desde las teorías de Piaget y Vygotsky. Liberabit, 23(1), https://doi.org/10.24265/liberabit.2017.v23n1.10 137-148.

Reiff, J. (1992). Learning Styles. What Research Says to the Teacher Series. ERIC. https://eric.ed.gov/?id=ED340506
Rodríguez, R. (2017). Los modelos de aprendizaje de Kolb, Honey y Mumford: implicaciones para la educación en ciencias. Sophia-Educación, 14 (1), 3-4. http://dx.doi.org/10.18634/sophiaj.14v.1i.698

Rodríguez, M., Peña, J. y García, O. (2016). Estudio cualitativo de las diferencias de género en la elección de opciones académicas en los estudiantes del bachillerato científicotécnico. Revista Interuniversitaria, 28(1), 189-207. http://dx.doi.org/10.14201/teoredu2016281189207

Romero, J., Romero, R. y Barboza, L. (2021). Programa instruccional basado en la neurociencia para mejorar el aprendizaje en los estudiantes universitarios. Revista San Gregorio. $46 \quad$ (16-29) ISSN 1390-7247. http://www.revista.sangregorio.edu.ec/index.php/REVIS TASANGREGORIO/article/view/1625

Sandoval, P., Rodríguez, F. y Maldonado, A. (2017). Evaluación de la alfabetización digital y pedagógica en TIC, a partir de las opiniones de estudiantes en Formación Inicial Docente. Educação e Pesquisa, 43(1), 127-143. https://www.redalyc.org/pdf/298/29849949009.pdf

SIES (junio del 2018). Informe Matrícula 2018 en Educación Superior en Chile. Ministerio de Educación. https://analisis.umag.cl/documentos/informe_matricula 2018_sies.pdf

Squire, L., Genzel, L., Wixted, J. y Morris, R. (2015). Memory Consolidation. Cold Spring Harbor Perspective in Biology,7(8) 10.1101/cshperspect.a021766

Varguillas, C. y Bravo, P. (2020). Virtualidad como herramienta de apoyo a la presencialidad: Análisis desde la mirada estudiantil. Revista de Ciencias Sociales (RCS), 26(1), 219-232. Recuperado de: https://dialnet.unirioja.es/servlet/articulo? codigo $=73844$ 16

Van Kesteren, M.T.R. y Meeter, M. (2020). How to optimize knowledge construction in the brain. npj Science of Learning - Nature, 5, 5. https://doi.org/10.1038/s41539020-0064-y

Vielma, E. y Salas, M. (2000). Aportes de las teorías de Vygotsky, Piaget, Bandura y Bruner. Paralelismo en sus posiciones en relación con el desarrollo. Educere, 3(9), 30-37. https://www.redalyc.org/pdf/356/35630907.pdf

Vogel, S. y Schwabe, L. (2016). Learning and memory under stress: implications for the classroom. npj Science of $\begin{array}{llll}\text { Learning } & - & \text { Nature, }\end{array}$ https://doi.org/10.1038/npjscilearn.2016.11

Vygotsky, L. (1979). El desarrollo de los procesos psicológicos superiores. Crítica, 94-138-139. https://saberespsi.files.wordpress.com/2016/09/vygostkiel-desarrollo-de-los-procesos-psicolc3b3gicossuperiores.pdf

Woolfolk, A. (2014). Diferencias de habilidad y enseñanza. En Prentice Hall, Quintanar (Ed.), Psicología educativa. México: Pearson Educación, pp. 128. https://saberespsi.files.wordpress.com/2016/09/psicologi a-educativa-woolfolk-7c2aa-edicion.pdf 